\title{
The Role of the Passive Voice Mindset in Regulating Healthy Eating
}

\author{
Ibrahim Senay ${ }^{1}$, Muhammet $\mathrm{Usak}^{2} \&$ Zeynep Ceren Acarturk ${ }^{1}$ \\ ${ }^{1}$ Department of Psychology, Istanbul Sehir University, Istanbul, Turkey \\ ${ }^{2}$ Science Education Center, Ankara, Turkey \\ Correspondence: Ibrahim Senay, Department of Psychology, Istanbul Sehir University, Istanbul, Turkey. E-mail: \\ ibrahimsenay@sehir.edu.tr
}

Received: February 16, 2017

Accepted: February 26, 2017

Online Published: March 9, 2017

doi:10.5539/ijps.v9n2p37

URL: http://doi.org/10.5539/ijps.v9n2p37

\begin{abstract}
Talking about eating in the passive, as opposed to the active voice, (e.g., The cake will be eaten vs. I will eat the cake) can lead people to see the act of eating to be triggered by the food to a greater extent, leading to the continuation of past eating habits. Depending on whether or not the past habits are healthy, the motivation for healthy eating may change as a result. In study 1 , writing passive sentences increased the motivation for healthy eating to the extent that people reported eating healthy in the past. Moreover, in study 2 across 127 languages spoken in 94 countries, when the acted-upons of actions (e.g., the food in the act of eating) became relatively more salient in a language, people became more likely to act on cultural habits that may be relatively healthier, decreasing unhealthy eating. The results are important for understanding the perceived role of food in starting eating as it impacts healthy eating across cultures.
\end{abstract}

Keywords: cross-cultural health, language, motivation, passive voice

\section{Introduction}

\subsection{Cross-Cultural Differences in Eating}

One important cross-cultural difference in eating behaviors concerns the degree to which eating is about the food vs. the goals of the people who engage in eating. Japanese, for example, are more likely than Americans to eat in response to environmental cues of food such as food products at the supermarket or cafeteria counters (Hawks, Madanat, Merrill, Goudy, \& Miyagawa, 2003). Americans, on the other hand, are relatively more likely to engage in eating as an additional activity such as eating while watching TV. Americans are also more likely than French and Belgians to see eating as an occasion to activate and pursue health goals while French and Belgians usually engage in eating for pleasure purposes (Rozin, Fischeler, Imada, Sarubin, \& Wrzesniewski, 1999; Rozin, 2005). Moreover, cultures are differentially motivated to use eating for goal-directed purposes such as showing differences in how often they eat to emotionally feel better or to socialize (Luomala, Sirieix, \& Tahir, 2009).

\subsection{The Passive Voice Mindset and Eating}

While the actual role of food (vs. people) in starting eating can change from culture to culture, the perceived role of the food (vs. people) in starting eating may change from person to person. One way in which this can happen may have to do with how people may want to talk about actions like eating. One of the important ways in which language as an important cultural tool can change our thoughts about actions like eating is to reverse how we usually think of the causal structure of such actions. We usually think of actors as the causes of actions such as focusing on the man in the sentence The man eats the cake as the cause of the observed action. However, in a language such as English we can also use a relatively less default option, the passive voice, and say The cake is eaten by the man. This would imply that the acted-upon, the cake, is relatively more responsible for the observed behavior, and that the act of eating is controlled by the actor, the man, to a lesser extent. In sum, the perceived role of food in starting eating is likely to be affected by the use of language in talking about events. As a result, not only the reported (Hawks et al., 2003; Luomala et al., 2009; Rozin et al., 1999; Rozin, 2005) but also the perceived role of food in starting eating may be important in determining cross-cultural patterns of eating.

When an inanimate/non-autonomous entity happens to be an actor as in the case of a brick hitting a man, it becomes more common to form a passive sentence to talk about this event (The man was hit by the brick) (Harris, 1978). The actor of a passive sentence such as $X$ in the sentence $Y$ is eaten by $X$ is also rated to have less 
animacy/causal agency than the acted-upon of this sentence, $Y$ (Johnson, 1967). Moreover, people describe a rape incident in the passive voice if they hold the victim, the acted-upon, as opposed to the assailant, the actor, relatively more responsible for the incident (Bohner, 2001). What would be the effect of talking, hence thinking, about eating in the passive voice (e.g., The cake will be eaten) on eating behaviors?

It is possible that the passive voice will shift our attention away from our role as actors in executing behaviors (e.g., The cake will be eaten vs. I will eat the cake) and to the acted-upon, the food in the case of eating (Harris, 1978; Johnson, 1967; Bohner, 2001). We might, thus, consider what foods are salient in our mind or what foods we often consume as a criterion to decide what we want to eat next. In Wendy and Neal's (2007) model of habitual behavior, for example, people rely on what they frequently do to infer what they want to do next. Thus, “.... a student with a habit to do homework on the computer after dinner may infer that the behavior reflects his or her strong academic achievement goals" (p. 854), leading the person to intentionally repeat this behavior later. Similarly, in Self Perception Theory, people infer the causes of their thoughts and attitudes indirectly from observing what they find themselves often doing (Bem, 1972). For example, if I often find myself eating cakes, I may infer that I like eating cakes. Given that we can better change our behaviors when we think of ourselves as actors as conceptualized in various behavior change models (Ajzen, 2002; Bandura, 1997; Deci \& Ryan, 2000), talking about behaviors in the passive, as opposed to active, voice (It will be done vs. I will do it) may reverse this tendency, leading us to continue our old habits. In sum, having a passive voice mindset may reverse our default tendency to focus on ourselves as the cause of what we want to eat, leading us to infer what we are motivated to eat from what we often eat. As a result, the motivation to continue habitual eating patterns (healthy or unhealthy) may increase.

Hypothesis 1. Leading people to think in the passive voice, which could be induced through the incidental exposure to passive sentences (supraliminal priming), will lead people to report stronger intentions for eating healthy to the extent that they report eating healthy in the past.

\subsection{The Passive Voice Mindset and Eating across Cultures}

Changes in world's diet habits away from traditional patterns of eating toward more sugar-, oil-, energy-dense and animal-source foods greatly contribute to the obesity pandemic all around the world in addition to the changing physical activity patterns (Popkin, 2005; Prentice, 2006). Given the generally unhealthy eating habits of today's societies, setting goals in line with one's contemporary eating habits may produce unhealthy eating styles. Thus, the salience of the passive voice mindset in a language, by motivating people to act on their habits, can lead to the continuation of unhealthy habits characterized by indulgence and the increased intake of fats and sugars.

However, habits are not just personal. They can also be cultural in the form of cultural traditions. The passive voice mindset may not just increase the tendency to act on personal habits but also the cultural ones. Given that relatively more traditional eating habits are usually healthier than relatively more contemporary eating styles (Popkin, 2005; Prentice, 2006), the passive voice mindset may also lead to healthier patterns of eating to the extent that it increases the reliance on more traditional habits.

\subsection{Measuring the Passive Voice Mindset and Its Impact Across Cultures}

In languages that mark the distinction between the passive and the active voice, the passive voice is not the default option. In these languages, therefore, the passive voice highlighting the perceived role of the food in starting eating may not be as salient as the active voice that highlights the perceived role of the people who eat. The passive voice may, however, become a relatively more salient option in a language when it is co-present with antipassives that exist in some languages other than English. Antipassives function to either completely hide the acted-upons of sentences such as turning the meaning of the sentence John eats the cake into the one similar to that of John eats, or make acted-upons seem to be indirectly involved in actions such as creating a meaning similar to that of John eats from the plate (Polinsky, 2013). When the passive voice is co-present with antipassives in a language, it is possible that the passive voice may become a relatively more salient option in speech for highlighting the role of acted-upons in actions (e.g., The cake is eaten vs. John eats). Previous studies have shown that the more frequent a linguistic feature is in a language, the more often the speakers of this language will use it to describe actions or events (Gennari, Sloman, Malt, \& Fitch, 2002; Slobin, 2003). Therefore, in languages that have both antipassive and passive sentences, these sentences will be more available to the speakers to represent events. The passive vs. the antipassive sentences will then be more frequently and likely to constitute two salient opposites to choose from when speakers describe events. 
One way to look at how the salience of the passive voice mindset in a culture will impact the cultural tendencies is to analyze how it is associated with the characterization of cultures along dimensions included in Hofstede's comprehensive model of national cultures (Hofstede G., Hofstede G. J., \& Minkov, 2010). These dimensions are determined based on the cross-cultural data collected from currently available 111 countries about the values of employees, students, managers and consumers living in these countries. Over the years the validity of the model in predicting behaviors in cultures has been well-established. Two of the dimensions of interest focused on in this study are the dimension of indulgence, indicating the motivation to engage in frequent behaviors such as habitual eating and the dimension of short vs. long-term orientation indicating the motivation to either continue or change past cultural habits, respectively.

Hypothesis 2a. Countries speaking languages that include both the passives and antipassives may have relatively higher scores on indulgence and a lower score on the long-term orientation dimension (i.e., the motivation to change cultural habits).

It is also possible to further analyze the link between the cultural dimensions and the indicators of healthy eating across countries reported by World Health Organization (2016) such as its data on the caloric intake values of countries.

Hypothesis $2 b$. In terms of eating outcomes, the indulgence facilitated by the passive voice mindset will lead to both healthy and unhealthy eating (i.e., lower vs. higher caloric intake) at the same time. To the extent that the salient passive voice increases the tendency to act on cultural habits that are usually healthier (decreased long-term orientation), it will lower caloric intake. To the extent that it does not lead to acting on cultural habits it will increase unhealthy eating.

\section{Study 1}

\subsection{Method}

\subsubsection{Participants}

One-hundred college students ( 72 females, $M=22.0$ for age) who were Native Speakers of Turkish and gave their consent to participate partook in the experiment in return for a course credit. Fifty people were randomly assigned to each of the two study conditions.

\subsubsection{Measures}

\subsubsection{Behavior Frequency}

The baseline frequency of healthy eating was measured on a 1-to-7 scale by one question asking how often one avoids foods high in fats or sugars.

\subsubsection{Goals}

For healthy eating, a single question asking whether the participant sometimes diets was used. The answer had a yes-or-no choice. The validity of this one-item measure was previously established (see e.g., Fishbach \& Shah, 2006).

\subsubsection{Motivation/Intention for Behavior}

To measure the intention and the motivation for eating heathy, Treatment Self-Regulation Questionnaire (Williams, Grow, Freedman, Ryan, \& Deci, 1996) developed within the framework of Self-Determination Theory (Deci \& Ryan, 2000) was included on the post-experiment questionnaire. The intention for, as well as the intrinsic (autonomous), extrinsic (controlled) and the lack of, motivation (amotivation) for healthy eating were reported on a 1-to-7 scale. All items were translated into Turkish in agreement by two native speakers of Turkish who were experts in English.

To establish the validity of the translated TSRQ, we first conducted a Parallel Analysis, a procedure based on Monte Carlo methods, by using O'Connor's (2000) procedure. Based on 5,000 simulated data sets, only the eigenvalues of the two highest factors fit the eigenvalues obtained from simulation. The eigenvalue of the third highest factor (eigenvalue $=1.18$ ) were outside the $95 \%$ CI of the simulated eigenvalues $[1.42,1.53]$, suggesting a 2-factor solution to be the most fitting. Second, we conducted a Confirmatory Factor Analysis with the original three factors: intrinsic motivation, extrinsic motivation, and amotivation. The three-factor model produced a poor structural fit, $\chi^{2} / \mathrm{df} .=2.65, G F I=.75, C F I=.73$, PCLOSE $<.001, R M S E A=.131$. After dropping the amotivation factor that had relatively smaller item weights as well as a weak item from the extrinsic motivation subscale, a two-factor model with the intrinsic and the modified extrinsic motivation produced a better fit, $\chi^{2} / \mathrm{df}$. 
$=1.64, G F I=.88, C F I=.94, P C L O S E=.073$, RMSEA $=.082$. The respective Cronbach's alpha values were 0.85 and 0.71 for the resulting intrinsic and extrinsic motivation subscales, indicating a good and an acceptable internal consistency, respectively. The amotivation subscale was not included in further analyses presented below.

\subsubsection{Procedure}

Participants were led to believe that they were going to complete a series of three separate studies. The first study was presented as a short study investigating the prevalence of various health and safety behaviors among college students. In this part, participants answered questions about their frequency of, and goal-directed attempts for, healthy eating as well as other filler behaviors such as exercising, smoking and obeying traffic rules. Then, as part of a seemingly separate study presented as an investigation of attitudes toward hand-writing, participants were randomly assigned to hand-write either the sentence $I$ will bring it or It will be brought in Turkish (getirilecek vs. getireceğim, trans.) for 15 times. After the hand writing-task, participants answered questions presumably measuring how much they enjoyed their hand-writing experience. Lastly, they reported their intention and reasons for heathy eating as part of a third study presented as an investigation of college students' future health goals.

\subsection{Results}

\subsubsection{Frequency of Behavior}

Diet intentions were submitted to an ANCOVA with sentence voice (passive vs. active voice) as a fixed factor and the baseline frequency of dieting (frequency of behavior) as a covariate by fully crossing the two factors. There was no significant effect of sentence voice, $F(1,96)=2.67, p=.105$. The diet intentions were stronger in the passive as compared to the active-voice condition, though in a statistically non-significant way $(M \mathrm{~s}=5.1 \mathrm{vs}$. 4.88 and $S D \mathrm{~s}=1.15$ vs. 1.26). However, as predicted and shown in Figure 1, the more frequently people dieted in the past, the stronger the intention to diet became as a result of the exposure to passive (vs. active) sentences, $F(1,96)=4.46, p=.037, \eta^{2}=.04$. Frequency of dieting increased intentions only in the passive voice condition, $F(1.48)=4.38, p=.042, \eta^{2}=.08$. It did not change intentions in the active-voice condition, $F(1,48)=1.14$, $p>.250$.

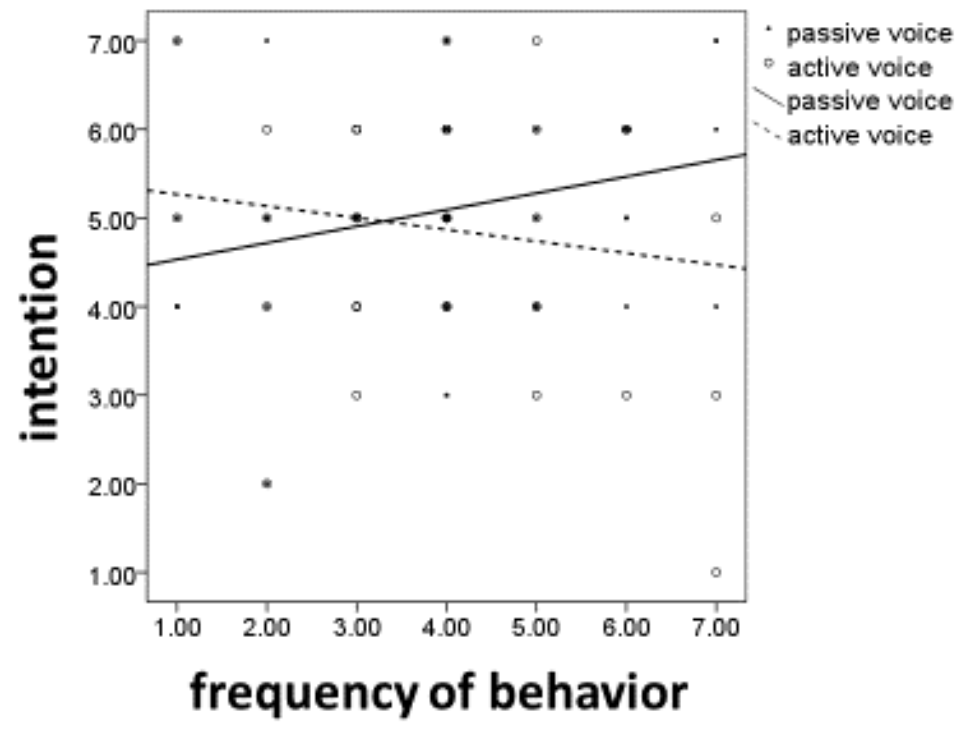

Figure 1. Scatterplots and best-fit lines of intention to diet as a function of the frequency of dieting in the passive voice group only or the active-voice group only, overlaid on a single graph

\subsubsection{Past Goals}

Diet intentions were submitted to an ANCOVA by fully crossing the factors sentence voice (passive vs. active) and diet goal (present vs. absent). Having a diet goal strengthened intentions for dieting in general $(M \mathrm{~s}=5.33 \mathrm{vs}$. 4.77 and $S D \mathrm{~s}=1.32$ vs. 1.07$), F(1,96)=5.51, p=.027, \eta^{2}=.05$. However, having a diet goal did not modify the 
effect of the passive voice on intention, $F(1,96)=1.24, p>.250$. This indicates that the effect of the passive voice concerns behavior frequency regardless of whether frequent behaviors are goal-directed or not.

\subsubsection{The Role of Intrinsic Motivation}

To model the mediation via motivation of the effect of the passive voice-by-frequency of behavior interaction on intention, we used Model 8 of Hayes' (2013) PROCESS procedure, which statistically tested the presence of a moderated mediation effect after controlling for the effect of the baseline diet goals. As shown in Figure 2, the passive voice decreased intrinsic motivation, $\beta=-1.07, t(93)=1.41, \Delta R^{2}=.05, \Delta F(1,93)=5.80, p=.018$. However, there was a significant passive voice-by-frequency of behavior interaction such that the more frequent the healthy eating at the baseline was, the more positive the effect of the passive voice on intrinsic motivation became, $\beta=.89, t(93)=2.00, \Delta R^{2}=.04, \Delta F(1,93)=4.01, p=.048$. When intrinsic motivation was included in the same model together with the passive voice-by-behavior frequency interaction as well as the lower-order component terms of this interaction to predict diet intentions, only the effect of intrinsic motivation remained significant, $\beta=.41, t(92)=4.25, \Delta R^{2}=.18, \Delta F(1,92)=18.07, p<.001$. The previously significant effect of the passive voice-by-behavior frequency interaction on diet intentions became non-significant, $\beta=.90, t(95)=2.04$, $\Delta R^{2}=.04, \Delta F(1,95)=4.15, p=.044$ vs. $\beta=.48, t(92)=1.14, \Delta R^{2}=.01, \Delta F(1,92)=1.30, p>.250$. The moderated-mediation was significantly different from zero only when intrinsic motivation was the mediator in the tested model, Moderated Mediation Index $=.16, S E=.09,95 \%$ CI [.01, 0.41]. A moderated mediation analysis with extrinsic motivation was not significant, Moderated Mediation Index $=-.01, S E=.03,95 \% \mathrm{CI}$ $[-.12,0.2]$.

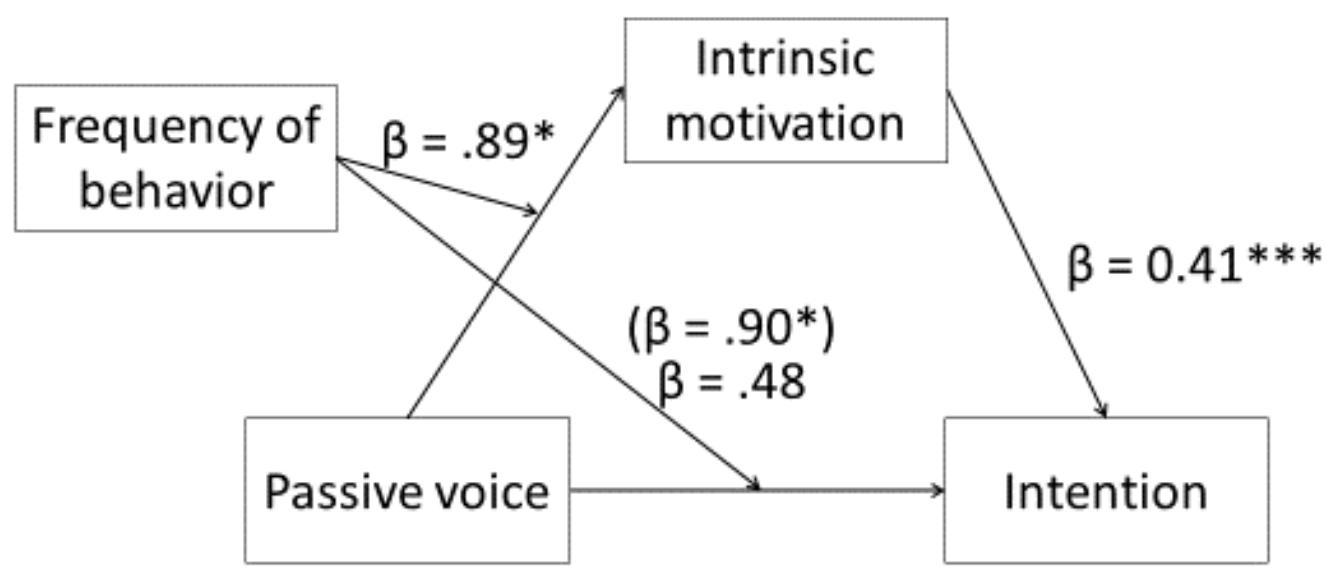

Figure 2. The moderated mediation of the effect of the passive voice on intention. All paths adjusted for baseline diet goals

$*: p<.05: * *: p<001$

\subsection{Discussion}

In Study 1, the frequency of dieting increased the positive effect of the passive voice on intrinsic motivation. As a result, intentions became stronger for frequent health behaviors that were not necessarily goal-directed. Thus, the passive voice mindset has the potential to turn habit-like, frequent diet behaviors into intrinsically motivated acts, helping maintain eating habits across time and contexts. The next study explored the consequences of this effect for healthy eating habits across world cultures.

\section{Study 2}

Because the default option for representing behaviors in a language that marks the active vs. the passive voice distinction is the active voice, the mere presence of the sentence (passive/active) voice in a language increases the salience of the active voice (Bohner, 2001; Harris, 1978; Johnson, 1967). However, the presence in a language of antipassive constructions that can be contrasted with the passive voice [The cake is eaten vs. John eats from (the plate)] can increase the relative salience of the passive voice for thinking about the role of acted-upons (e.g., the cake) in causing actions (e.g., eating) (Polinsky, 2013). As a result, a 2 x 2 interaction was 
predicted between the factors antipassives and the sentence voice as they would affect eating-related outcomes. Specifically, we predicted that the passive-by-antipassive interaction will increase habitual behaviors, increasing indulgence. Indulgence, in turn, was expected to increase unhealthy eating (high fat and sugar intake), leading to a greater daily caloric intake. The passive voice mindset may not only increase the tendency to act on personal but also cultural habits, decreasing long-term orientation (i.e., motivation to change cultural habits). The relatively healthier patterns of eating in more traditional societies can, in turn, decrease the fat and sugar consumption, hence daily caloric intake. Thus, a sentence voice-by-antipassive interaction was expected, on one hand, to increase caloric intake through indulgence, while decreasing it through long-term orientation.

\subsection{Method}

\subsubsection{Measures}

We used The World Atlas of Language Structures database (Dryer \& Haspelmath, 2013) categorizing all world languages based on published linguistic evidence according to whether they include passive/active (Siewierska, 2013) or antipassive (Polinsky, 2013) constructions. We also used a comprehensive reference work cataloging all of the world's known living languages, Ethnologue: Languages of the World, to locate countries where each language identified by its ISO 639-3 code is spoken (About the Ethnologue, n.d.). Next, we used Hofstede's cultural dimensions (Hofstede et al., 2010) to identify where a country lies on each of the following cultural dimensions: individualism (i.e., the degree of independence maintained among the members of a society), masculinity (i.e., wanting to be the best vs. liking what one does), uncertainty avoidance (i.e., the degree of being threatened by unknown situations), power distance (i.e., the degree of accepting the unequal distribution of power in a society), long-term orientation (i.e., changing vs. keeping cultural traditions) and indulgence (i.e., having low vs. high control over one's impulses). Lastly, we used World Health Organization Global Database (World Health Organization Global Database on Body Mass Index, 2016) to obtain information about countries' daily caloric intake from fats and sugars as well as total daily caloric intake.

\subsection{Results}

\subsubsection{The Effect of Sentence Voice and Antipassives}

Because countries speaking a particular language may be similar to one another due to reasons other than language, we used Linear Mixed Models with a compound symmetry covariance matrix to analyze between 82 and 94 countries as embedded within 119 to 127 languages in models predicting different cultural dimensions as included in Hofstede's model. As expected and shown in Figure 3, the sentence voice-by-antipassive interaction was only significant for long-term orientation and indulgence, $F(1,142.57)=4.41, p=.037, \omega^{2}=.03$, and, $F(1,132.57)=5.85, p=.017, \omega^{2}=.02$, respectively. Planned contrasts showed that the presence of sentence voice in a language decreased indulgence $(M \mathrm{~s}=44.92$ vs. $56.97, S E \mathrm{~s}=2.59$ vs. 3.63$)$ only if the language did not have antipassive constructions, $t(113.1)=2.704, p=.008, g=.56,95 \%$ CI $[.15, .97]$. When the language had antipassive constructions, the presence of sentence voice had a non-significant tendency to increase indulgence, instead $(M \mathrm{~s}=67.42$ vs. $55.01, S E \mathrm{~s}=7.30$ vs. 5.39$), t(137.79)=1.367, p=.164$. Similarly, the presence of sentence voice increased long-term orientation $(M \mathrm{~s}=44.25$ vs. $33.34, S E \mathrm{~s}=2.6$ vs. 3.56$)$ only if antipassives were absent in a language, $t(128.536)=2.472, p=.015, g=0.51,95 \%$ CI $[.10, .91]$. When antipassives were present in a language, sentence voice had a non-significant tendency to decrease long-term orientation $(M \mathrm{~s}=30.82$ vs. $40.96, S E \mathrm{~s}=7.28$ vs. 5.29$), t(146.18)=1.127, p>.250$. In sum, when the passive voice is contrasted with antipassives hence becomes more salient as a mindset in a language, long-term orientation relatively decreases and indulgence relatively increases in countries speaking this language. There was no significant sentence voice-by-antipassive interaction for power distance, individualism, masculinity, or uncertainty avoidance, $F(1,206.49)=.024, p>.250, F(1,112.82)=0.470, p>.250, F(1,219.26)=.289$, $p>.250, F(1,153.69)=.348, p>.250$, respectively. 


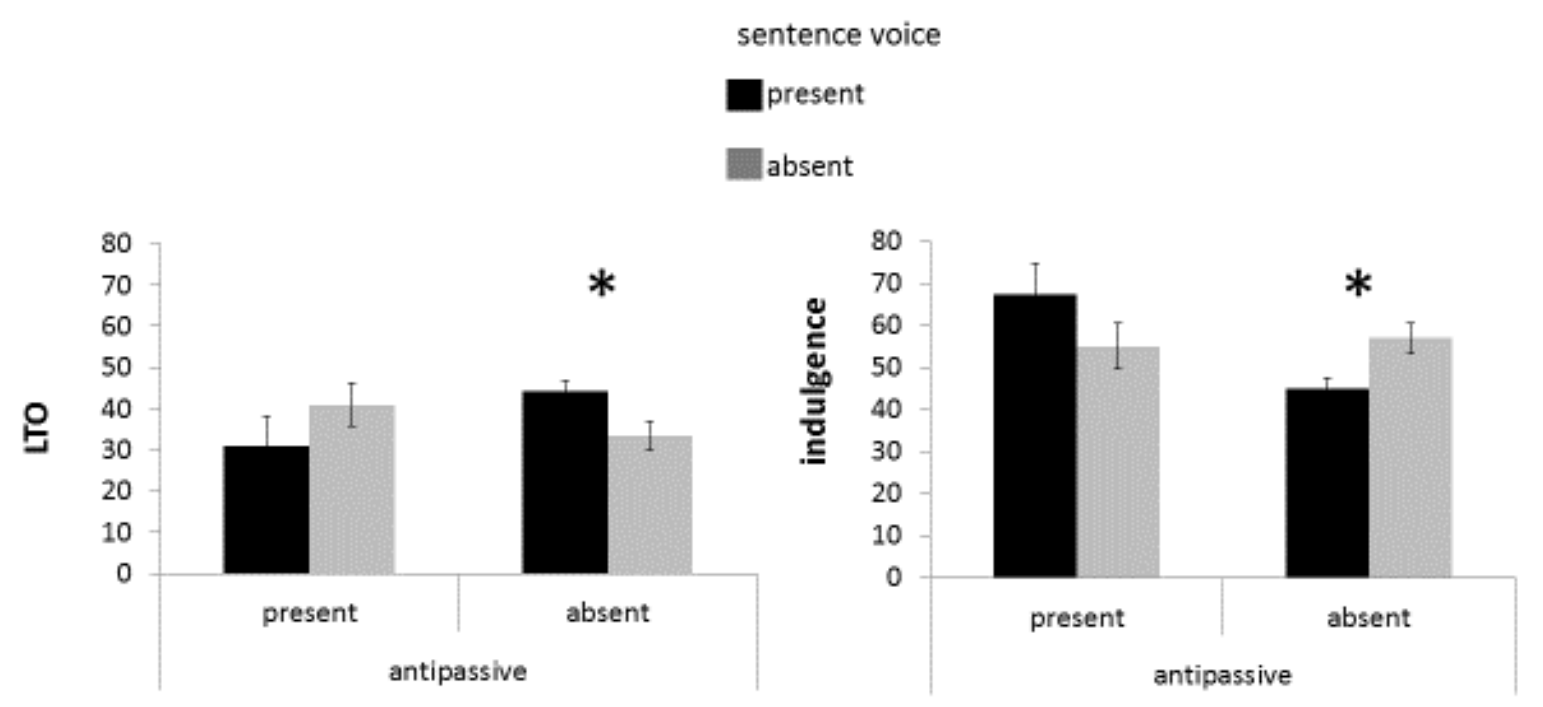

Figure 3. Long-Term Orientation (LTO) and indulgence scores of countries with error bars as a function of the presence vs. absence of passive/active vs. antipassive constructions in country languages

\subsubsection{Mediating Role of Indulgence and Long Term Orientation in Caloric Intake}

In regression analyses of averaged country scores calculated for each language, indulgence and long-term orientation mediated the effect of the sentence voice-by-antipassive interaction on eating behaviors in a multiple serial mediation analysis based on Hayes' Model 6 (2013). The passive-by-antipassive interaction was entered as the independent variable while keeping the lower-order terms of this interaction (the presence of active/passives and the presence of antipassives) as covariates in all tested models. The total daily caloric intake was entered as the outcome variable. Indulgence, long-term orientation and daily calories from fats and sugars were entered as the three mediating factors. All other cultural dimensions (uncertainty avoidance, individualism, power distance and masculinity) were entered as covariates in all models. There was no direct effect of the passive voice-by-antipassive interaction on total daily caloric intake, Direct Effect $b=25.28 S E=87.30$, 95\% CI [-147.86, 198.42]. The overall indirect effects of the sentence voice-by-antipassive interaction on total caloric intake was also not significant, Indirect Effect $b=211.17 S E=128.71,95 \%$ CI [-48.05, 470.08]. This lack of a significant effect was due to the two opposite effects of the passive-by-antipassive interaction on caloric intake.

As shown in Figure 4, the interaction between the sentence voice and antipassives significantly increased indulgence, $\beta=.27, t(106)=2.34, p=.007, \Delta R^{2}=.04, \Delta F(1,107)=7.49, p=.007$. Indulgence, in turn, had two different effects on the caloric intake from fats and sugars. On one hand, indulgence decreased long-term orientation, $\beta=-.59, t(105)=6.36, p<.001, \Delta R^{2}=.20, \Delta F(1,106)=40.42, p<.001$. Long-term orientation, in turn, increased daily calories taken from fats and sugars, $\beta=.31, t(108)=4.43, p<.001, \Delta R^{2}=.05, \Delta F(1,109)=$ $19.65, p<.001$. Thus, indulgence had a negative effect on the daily calories from fats and sugars through long-term orientation. On the other hand, indulgence directly increased daily calories from fats and sugar, $\beta$ $=.29, t(108)=3.80, p<.001, \Delta R^{2}=.04, \Delta F(1,109)=14.46, p<.001$. Daily calories from fats and sugars increased the total daily caloric intake, $\beta=.84, t(107)=11.21, p<.001, \Delta R^{2}=.19, \Delta F(1,108)=125.76, p$ $<.001$. Long-term orientation also directly increased daily caloric intake, $\beta=.16, t(107)=2.52, p=.008, \Delta R^{2}$ $=.01, \Delta F(1,108)=7.39, p=.008$. The negative effect of the salient passive voice (i.e., the sentence voice-by-antipassive interaction) on caloric intake through first indulgence and then long-term orientation was significant, Indirect Effect $b=-42.04, S E=35.11,95 \%$ CI $[-172.75,-8.20]$. The negative effect of the salient passive voice on caloric intake through first indulgence, then long-term orientation, and then lastly through daily calories from fats and sugars was also significant, Indirect Effect $\mathrm{b}=-36.08, S E=27.84,95 \%$ CI [-136.30, -6.87]. Finally, the positive effect of the salient passive voice on caloric intake through first indulgence, and then through daily calories taken from fats and sugars was significant, Indirect Effect $b=148.07, S E=66.83,95 \% \mathrm{CI}$ [58.60, 366.68]. 


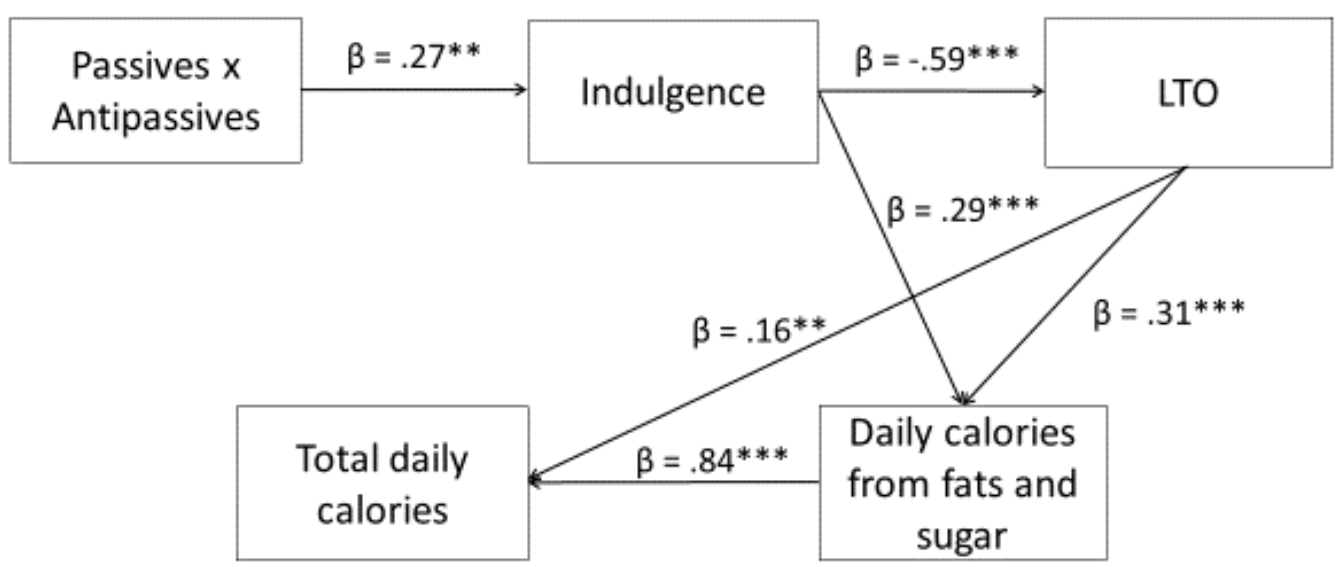

Figure 4. The combinations of significant mediations via indulgence, Long-Term Orientation (LTO), and daily calories from fats and sugar of the effect of the sentence voice-by-antipassive interaction on the total daily caloric intake. All paths are adjusted (see the text)

${ }^{* *}: p<.01 ; * * *: p<.001$

\subsubsection{Other Effects}

The mere presence of antipassives in a language increased individualism in countries speaking this language $(M \mathrm{~s}$ $=44.2$ vs. $60.4, S E \mathrm{~s}=2.9$ vs. 5.5$), F(1,112.82)=6.89, p=.01, \omega^{2}=.04$. This shows that the passive voice's effect on long-term orientation and indulgence was exclusively due to sentence voice rather than antipassives, which additionally increased individualism, a dimension, which was unaffected by sentence voice. There was no other interaction or main effect for any other cultural dimension.

\subsection{Discussion}

When the passive voice in a language becomes a relatively more salient option for highlighting the role of acted-upons (vs. actors) in events, it can increase unhealthy eating and caloric intake in cultures speaking this language by increasing indulgence. At the same time, the passive voice may decrease long-term orientation, ultimately decreasing the intake of fats and sugars and total daily caloric intake. The results also showed that the presence of antipassives in a language can increase individualism, a dimension, which was not affected by the presence of sentence voice in a language. Therefore, the interaction effect between the sentence voice and the antipassives on indulgence and long-term orientation seem to specifically and primarily concern the sentence voice rather than antipassives. The presence of antipassives in a language seems to only modify the effect of the sentence voice on the predicted outcomes of Study 2.

Thus, the beneficial effects of the salient passive voice in a language of a society depends on whether it will ultimately lead to the maintenance of cultural habits or not. As similar to the results obtained from Study 1, the passive voice mindset in Study 2 increased the tendency to act on habits (i.e., indulgence) in general. When the passive voice mindset also led to acting on cultural habits that may be relatively healthier (i.e., short-term orientation), the eating behaviors became healthier.

\section{General Discussion}

Over two studies and 127 languages, the passive voice mindset motivated people to set goals in line with their habits by redirecting their attention to the role of the acted-upons like food, as opposed the actors like people who eat, in starting actions like eating. As a result, to the extent that people had healthy eating habits (Study 1) or motivated to maintain their cultural habits (Study 2), the passive voice mindset led to healthier eating styles, decreasing the calories taken from fats and sugars and daily caloric intake across nations. Altogether, these findings suggest that the passive voice not only influences the interpretation of messages (Harris, 1978; Johnson, 1967; Bohner, 2001), but can also reverse our thought habits (McNeill \& Duncan, 2000; Slobin, 1996) that usually put actors (vs. acted-upons) in the center of actions (Ajzen, 2002; Bandura, 1997; Deci \& Ryan, 2000). As a result, frequent habits that are not necessarily goal-directed can be turned into intrinsically motivated acts, leading to the maintenance of frequent behaviors such as healthy eating habits and lifestyles across time, contexts and cultures. 
Previous studies identified cross-cultural differences in how much people's goals vs. food cues play a role in starting eating (Hawks et al., 2003), how much people think of their goals vs. pleasure in eating (Rozin et al., 1999; Rozin, 2005) and how much people pursue emotional-regulation goals through eating (Luomala et al., 2009). The Americans as compared to Belgians, French and Japanese were, for example, reported to be more likely to engage in eating as a goal directed activity such as eating to stay healthy or eating as an additional activity to watching TV. The present study's results show that not only culture but also the mindset associated with spoken languages in a culture can change people's conceptualization of, and motivation for, eating. The perceived role of the food in starting eating may be changed by the use of language and may be relevant to understanding the cross-cultural patterns of eating. The languages that highlight the passive voice mindset may be both beneficial and risky for the formation of healthy eating habits in a society. It is only when people are eating healthy or a country population is motivated to continue their traditional patterns of behavior, the passive voice mindset may increase healthy eating.

Because the passive voice mindset led to indulgence across cultures in the present study, the results are also relevant for understanding the role of indulgence in healthy eating especially in countries that score high on indulgence such as USA. On one hand, indulgence motivated by the passive voice mindset led to unhealthier eating as would be normally expected. On the other, it led to a healthier style of eating with lower rates of consumed fats and sugars. This latter, healthier, effect of indulgence on eating was present to the extent that people in a country with higher scores of indulgence were also motivated to continue their cultural traditions that may include relatively healthier eating styles (Popkin, 2005; Prentice, 2006). Thus, the detrimental effects of indulgence on healthy eating may especially be the case in cultures that are not motivated to continue their traditional eating habits. Moreover, the passive voice mindset can be one of the ways to partially ensure that indulgence will decrease long-term orientation, ultimately producing healthy eating outcomes.

Talking about behaviors in ways to highlight either the role of actors (I can do it) or actions (Just do it) has been shown to be a powerful technique for controlling and motivating goal-directed behaviors (Hardy, 2006; Hatzigeorgiadis, Zourbanos, Galanis, \& Theodorakis, 2011). Using passive as opposed to active sentences to talk about behaviors (It will be done), for example, was shown to increase task performance on both motor and verbal regulation tasks (Senay, Usak, \& Prokop, 2015). That was because the passive voice mindset increased the taskas opposed self-focus, allowing for a better concentration on tasks. The present study findings show that the passive voice mindset may not just increase goal-directed but also habitual behaviors. Given that maintaining adaptive habits such as exercising and healthy eating is as important as setting a goal to change the maladaptive ones such as smoking (see, e.g., van't Riet, Sijtsema, Dagevos, \& De Bruijn, 2011), language in general, and the passive voice mindset in specific, may prove useful as a tool for ensuring the maintenance of adaptive behaviors across time and contexts in addition to providing the initial motivation for starting these adaptive behaviors.

In the present investigation the passive voice mindset was implicitly induced through a supraliminal priming procedure in Study 1. There is evidence to suggest that behaviors are better facilitated when people are explicitly trained to use language during their task performance (Hatzigeorgiadis et al., 2011). Thus, the passive voice mindset may be more effective when people explicitly use passive sentences while they engage in a target behavior. One area of future research may be to train people to form implementation intentions that have been shown to be an effective way to merge the gap between intention and behavior across a variety of contexts (Gollwitzer \& Sheeran, 2006). Such pre-formulated and linguistically expressed intentions about food cues (e.g., Whenever I encounter healthy food cues during my meals, I will say to myself "The food will be eaten") may be especially helpful in maintaining healthy eating among healthy eaters. In Stages of Change Model (Prochaska \& DiClementi, 1983), for example maintaining behaviors is a crucial stage in acquiring healthy habits. Forming intentions expressed in the passive voice may especially be helpful for individuals who have reached this stage in their behavior change efforts.

Another area of future research may be to test in other languages that have antipassives some of the effects reported here in Turkish. This may better reveal the function of antipassive sentences as they interact with passive sentences in ultimately shaping motivation. Further studies are, in general, needed to understand how thought habits instilled by different languages shape the way we habitually comprehend events as a whole (Zwaan \& Radvansky, 1998) with important consequences for motivating adaptive behaviors and lifestyles in various health, performance and cultural settings. 


\section{References}

About the Ethnologue. (n.d.). Retrieved May 23, 2015, from http://www.ethnologue.com/about

Ajzen, I. (2002). Perceived behavioral control, self-efficacy, locus of control and the theory of planned behavior. Journal of Applied Social Psychology, 32, 1-20. https://doi.org/10.1111/j.1559-1816.2002.tb00236.x

Bandura, A. (1997). Self-efficacy, the exercise of control. New York: W.H. Freeman.

Bem, D. J. (1972). Self-perception theory. In L. Berkowitz (Ed.), Advances in experimental social psychology (Vol. 6, pp. 1-62). New York: Academic Press. https://doi.org/10.1016/s0065-2601(08)60024-6

Bohner, G. (2001). Writing about rape: use of the passive voice and other distancing text features as an expression of perceived responsibility of the victim. British Journal of Social Psychology, 40, 515-529. https://doi.org/10.1348/014466601164957

Deci, E. L., \& Ryan, R. M. (2000). The "what" and "why" of goal pursuits: Human needs and the self-determination of behavior. Psychological Inquiry, 11(4), 227-268. https://doi.org/10.1207/S15327965PLI1104_01

Dryer, M. S., \& Haspelmath, M. (2013). The World Atlas of Language Structures Online. Leipzig: Max Planck Institute for Evolutionary Anthropology.

Fishbach, A., \& Shah, J. Y. (2006). Self-control in action: Implicit dispositions toward goals and away from temptations. Journal of Personality and Social Psychology, 90, 820-832. https://doi.org/10.1037/0022-3514.90.5.820

Gennari, S. P., Sloman, S. A., Malt, B. C., \& Fitch, W. T. (2002). Motion events in language and cognition. Cognition, 83, 49-79. https://doi.org/10.1016/S0010-0277(01)00166-4

Gollwitzer, P. M., \& Sheeran, P. (2006). Implementation intentions and goal achievement: A meta-analysis of effects and processes. Advances in Experimental Social Psychology, 38, 69-119. https://doi.org/10.1016/S0065-2601(06)38002-1

Hatzigeorgiadis, A., Zourbanos, N., Galanis, E., \& Theodorakis, Y. (2011). Self-talk and sports performance: A $\begin{array}{llll}\text { meta-analysis. Perspectives on Psychological Science, 6(4), 348-356. } & \text { on }\end{array}$ https://doi.org/10.1177/1745691611413136

Hardy, J. (2006). Speaking clearly: A critical review of the self-talk literature. Psychology of Sport and Exercise, 7, 81-97. https://doi.org/10.1016/j.psychsport.2005.04.002

Harris, M. (1978). Noun animacy and the passive voice: A developmental approach. Quarterly Journal of Experimental Psychology, 30, 495-504. https://doi.org/10.1080/00335557843000089

Hawks, S. R., Madanat, H. N., Merrill, R. M., Goudy, M. B., \& Miyagawa, T. (2003). A cross-cultural analysis of "motivation for eating" as a potential factor in the emergence of global obesity: Japan and the United States. Health Promotion International, 18, 153-162. https://doi.org/10.1093/heapro/18.2.153

Hayes, A. F. (2013). Introduction to Mediation, Moderation, and Conditional Process Analysis. New York: Guilford.

Hofstede, G., Hofstede, G. J., \& Minkov, M. (2010). Cultures and Organizations: Software of the Mind (Revised and Expanded 3rd ed.). New York: McGraw-Hill USA.

Hofstede, G., Neuijen, B., Ohayv, D. D., \& Sanders, G. (1990). Measuring Organizational Cultures: A Qualitative and Quantitative Study Across Twenty Cases. Administrative Science Quarterly, 35(2), 286-316. https://doi.org/10.2307/2393392

Johnson, M. G. (1967). Syntactic position and rated meaning. Journal of Verbal Learning and Verbal Behavior, 6, 240-246. https://doi.org/10.1016/S0022-5371(67)80103-8

Luomala, H. T., Sirieix, L., \& Tahir, R. (2009). Exploring Emotional-Eating Patterns in Different Cultures: Toward a Conceptual Framework Model. Journal of International Consumer Marketing, 21, 231-245. https://doi.org/10.1080/08961530802202818

MacKinnon, D. P., Krull, J. L., \& Lockwood, C. M. (2000). Equivalence of the mediation, confounding and suppression effect. Prevention Science, 1(4), 173-181. https://doi.org/10.1023/A:1026595011371 
McNeill, D., \& Duncan, S. D. (2000). Growth points in thinking-for-speaking. In D. McNeill (Ed.), Language and Gesture (pp. 141-161). Cambridge: Cambridge University Press. https://doi.org/10.1017/CBO9780511620850.010

O'Connor, B. P. (2000). SPSS and SAS programs for determining the number of components using parallel analysis and Velicer's MAP test. Behavior Research Methods, Instrumentation, and Computers, 32, 396-402. https://doi.org/10.3758/BF03200807

Prentice, A. M. (2006). The emerging epidemic of obesity in developing countries. International Journal of Epidemiology, 35, 93-99. https://doi.org/10.1093/ije/dyi272

Prochaska, J. O., \& DiClemente, C. C. (1983). Stages and processes of self-change of smoking: Toward an integrative model of change. Journal of Consulting and Clinical Psychology, 51(3), 390-395. https://doi.org/10.1037/0022-006X.51.3.390

Polinsky, M. (2013). Antipassive constructions. In M. S. Dryer, \& M. Haspelmath (Eds.), The World Atlas of Language Structures Online. Leipzig: Max Planck Institute for Evolutionary Anthropology.

Popkin, B. M. (2005). Using research on the obesity pandemic as a guide to a unified vision of nutrition. Public Health Nutrition, 8, 724-729. https://doi.org/10.1079/PHN2005776

Rozin, P. (2005). The meaning of food in our lives: A cross-cultural perspective on eating and well-being. Journal of Nutrition Education and Behavior, 37, S107-S112. https://doi.org/10.1016/S1499-4046(06)60209-1

Rozin, P., Fischeler, C., Imada, S., Sarubin, A., \& Wrzesniewski, A. (1999). Attitudes to food and the role of food in life in the U.S.A., Japan, Flemish Belgium and France: Possible implications for the diet-health debate. Appetite, 33, 163-180. https://doi.org/10.1006/appe.1999.0244

Senay, I., Usak, M., \& Prokop, P. (2015). Talking about behaviors in the passive voice increases task performance. Applied Cognitive Psychology, 29, 262-270. https://doi.org/10.1002/acp.3104

Siewierska, A. (2013). Passive constructions. In M. S. Dryer, \& M. Haspelmath (Eds.), The World Atlas of Language Structures Online. Leipzig: Max Planck Institute for Evolutionary Anthropology.

Slobin, D. I. (1996). Thinking for speaking. In J. Gumperz, \& S. Levinson (Eds.), Rethinking Linguistic Relativity (pp. 271-323). Cambridge University Press.

Slobin, D. I. (2003). Language and thought online: Cognitive consequences of linguistic relativity. In D. Gentler, \& S. Golden-Meadow (Eds.), Language in Mind: Advances in Study of Language and Thought (pp. 157-192). The MIT Press.

van't Riet, J., Sijtsema, S. J., Dagevos, H., \& Bruijn, G. D. (2011). The importance of habits in eating behavior: An overview and recommendations for future research. Appetite, 57, 585-596. https://doi.org/10.1016/j.appet.2011.07.010

Wendy, W., \& Neal, D. T. (2007). A new look at habits and the habit-goal interface. Psychological Review, 114(4), 843-863. https://doi.org/10.1037/0033-295X.114.4.843

Williams, G. C., Grow, V. M., Freedman, Z. R., Ryan, R. M., \& Deci, E. L. (1996). Motivational predictors of weight loss and weight-loss maintenance. Journal of Personality and Social Psychology, 70, 115-126. https://doi.org/10.1037/0022-3514.70.1.115

World Health Organization Global Database on Body Mass Index. (2016). Retrieved from http://apps.who.int/bmi/index.jsp

Zwaan, R. A., \& Radvansky, G. A. (1998). Situation models in language comprehension and memory. Psychological Bulletin, 123, 162-185. https://doi.org/10.1037/0033-2909.123.2.162

\section{Copyrights}

Copyright for this article is retained by the author(s), with first publication rights granted to the journal.

This is an open-access article distributed under the terms and conditions of the Creative Commons Attribution license (http://creativecommons.org/licenses/by/4.0/). 